\title{
Coherent transport of neutral atoms in spin-dependent optical lattice potentials
}

\author{
Olaf Mandel, Markus Greiner, Artur Widera, Tim Rom, Theodor W. Hänsch, and Immanuel Bloch* \\ Ludwig-Maximilians-Universität, Schellingstr. 4/III, 80799 Munich, Germany \\ Max-Planck-Institut für Quantenoptik, 85748 Garching, Germany
}

(Dated: September 3, 2018)

\begin{abstract}
We demonstrate the controlled coherent transport and splitting of atomic wave packets in spindependent optical lattice potentials. Such experiments open intriguing possibilities for quantum state engineering of many body states. After first preparing localized atomic wave functions in an optical lattice through a Mott insulating phase, we place each atom in a superposition of two internal spin states. Then state selective optical potentials are used to split the wave function of a single atom and transport the corresponding wave packets in two opposite directions. Coherence between the wave packets of an atom delocalized over up to 7 lattice sites is demonstrated.
\end{abstract}

Over the last years Bose-Einstein condensates in optical lattices have opened fascinating new experimental possibilities in condensed matter physics, atomic physics, quantum optics and quantum information processing. Already now the study of Josephson junction like effects [1, 2], the formation of strongly correlated quantum phases [3, 4, 5] and the observation of the collapse and revival of the matter wave field of a BEC 6 ] have shown some of these diverse applications. In an optical lattice, neutral atoms can be trapped in the intensity maxima (or minima) of a standing wave light field due to the optical dipole force 7 ]. So far the optical potentials used have been mostly independent of the internal ground state of the atom. However, it has been suggested that by using spin-dependent periodic potentials one could bring atoms on different lattice sites into contact and thereby realize fundamental quantum gates [8, 9, 10, 11], create large scale entanglement [12, 13], excite spin waves 14], study quantum random walks [15] or form a universal quantum simulator to simulate fundamental complex condensed matter physics hamiltonians 16]. Here we report on the realization of a coherent spin-dependent transport of neutral atoms in optical lattices [17]. We show how the wave packet of an atom that is initially localized to a single lattice site can be split and delocalized in a controlled and coherent way over a defined number of lattice sites.

In order to realize a spin dependent transport for neutral atoms in optical lattices, a standing wave configuration formed by two counterpropagating laser beams with linear polarization vectors enclosing an angle $\theta$ has been proposed [8, 12]. Such a standing wave light field can be decomposed into a superposition of a $\sigma^{+}$ and $\sigma^{-}$polarized standing wave laser field, giving rise to lattice potentials $V_{+}(x, \theta)=V_{0} \cos ^{2}(k x+\theta / 2)$ and $V_{-}(x, \theta)=V_{0} \cos ^{2}(k x-\theta / 2)$. Here $k$ is the wave vector of the laser light used for the standing wave and $V_{0}$ is the potential depth of the lattice. By changing the polarization angle $\theta$ one can thereby control the separation between the two potentials $\Delta x=\theta / 180^{\circ} \cdot \lambda_{x} / 2$. When increasing $\theta$, both potentials shift in opposite directions and overlap again when $\theta=n \cdot 180^{\circ}$, with $n$ being an integer. For a spin-dependent transfer two in- ternal spin states of the atom should be used, where one spin state dominantly experiences the $V_{+}(x, \theta)$ potential and the other spin state mainly experiences the $V_{-}(x, \theta)$ dipole force potential. Such a situation can be realized in rubidium by tuning the wavelength of the optical lattice laser to a value of $\lambda_{x}=785 \mathrm{~nm}$ between the fine structure splitting of the rubidium D1 and D2 transition. Then the dipole potential experienced by an atom in e.g. the $|1\rangle \equiv\left|F=2, m_{F}=-2\right\rangle$ state is given by $V_{1}(x, \theta)=V_{-}(x, \theta)$ and that for an atom in the $|0\rangle \equiv\left|F=1, m_{F}=-1\right\rangle$ state is given by $V_{0}(x, \theta)=3 / 4 V_{+}(x, \theta)+1 / 4 V_{-}(x, \theta)$. If an atom is now first placed in a coherent superposition of both internal states $1 / \sqrt{2}(|0\rangle+i|1\rangle)$ and the polarization angle $\theta$ is continuously increased, the spatial wave packet of the atom is split with both components moving in opposite directions. In a fixed polarization configuration, spindependent tunnelling between two lattice sites has been observed in [18].

As in our previous experiments, Bose-Einstein condensates of up to $3 \times 10^{5}$ atoms are created in the $\left|F=1, m_{F}=-1\right\rangle$ hyperfine state in a harmonic magnetic trap with almost isotropic oscillation frequencies of $\omega=2 \pi \times 16 \mathrm{~Hz}$. A three dimensional lattice potential is then superimposed on the Bose-Einstein condensate and the intensity raised in order to drive the system into a Mott insulating phase [5] and thereby localize the atoms to individual lattice sites. Two of the three orthogonal standing wave light fields forming the lattice potential are operated at a wavelength of $\lambda_{y, z}=840 \mathrm{~nm}$. For the third standing wave field along the horizontal $x$-direction a laser at a wavelength of $\lambda_{x}=785 \mathrm{~nm}$ is used. Along this axis a quarter wave plate and an electro-optical modulator (EOM) allow us to dynamically rotate the polarization vector of the retro-reflected laser beam through an angle $\theta$ by applying an appropriate voltage to the EOM (see Fig.11). Initially the polarization angle $\theta$ is set to a lin\|lin polarization configuration. After reaching the Mott insulating phase we completely turn off the harmonic magnetic trapping potential but maintain a $1 \mathrm{G}$ homogeneous magnetic field along the $x$-direction in order to preserve the spin polarization of the atoms. This 
homogeneous field is actively stabilized to an accuracy of $\approx 1 \mathrm{mG}$. Shortly before moving the atoms along this standing wave direction we adiabatically turn off the lattice potentials along the $y$ - and $z$-direction. This is done in order to reduce the interaction energy, which strongly depends on the confinement of the atoms at a single lattice site. We can thereby study the transport process itself, without having to take into account the phase shifts in the many body state that result from a coherent collisional interaction between atoms.

By using microwave radiation around $6.8 \mathrm{GHz}$ we are able to drive Rabi oscillations between the $|0\rangle$ and the $|1\rangle$ state with resonant Rabi frequencies of $\Omega=2 \pi \times 40 \mathrm{kHz}$, such that e.g. a $\pi$-pulse can be achieved in a time of $12.5 \mu \mathrm{s}$. The microwave field therefore allows us to place the atom in any of the two internal states $|0\rangle$ or $|1\rangle$ or an arbitrary superposition of both states.

During the shifting process of the atoms it is crucial to avoid unwanted vibrational excitations, especially if the shifting process would be repeated frequently. We therefore analyze the timescale for such a movement process in the following way. First the atom is placed either in state $|0\rangle$ or state $|1\rangle$ by using microwave pulses in a standing wave lin |lin polarization configuration. Then we rotate the polarization to an angle $\theta=180^{\circ}$ in a linear ramp within a time $\tau$, such that again a lin\|lin polarization configuration is achieved. However, during this process the atoms will have moved by a distance $\pm \lambda_{x} / 4$ depending on their internal state. In order to determine whether any higher lying vibrational states have been populated, we adiabatically turn off the lattice potential within a time of $500 \mu \mathrm{s}$. The population of the energy bands is then mapped onto the population of the corresponding Brillouin zones [19, 20]. By counting the number of atoms outside of the first Brillouin zone of the system relative to the total number of atoms we are able to determine the fraction of vibrationally excited atoms after the shifting of the lattice potential (see Fig. 2). For a perfectly linear ramp with infinite acceleration at the beginning and ending of the ramp one would expect the fraction of atoms in the first vibrational state to be given by $\left|c_{1}(\tau)\right|^{2}=2 v^{2} /\left(a_{0} \omega\right)^{2} \sin ^{2}(\omega \tau / 2)$, where $v=\lambda_{x} /(4 \tau)$ is the shift velocity, $a_{0}$ the size of the ground state harmonic oscillator wave function and $\omega$ the vibrational frequency on each lattice site.

We have measured the vibrational frequencies on a lattice site for different polarization angles $\theta$ by slightly modulating the lattice position and observing a resonant transfer of atoms to the first excited vibrational state. For atoms in the $|1\rangle$ state the vibrational frequencies remain constant for different polarization angles $\theta$ as the lattice potential depth $V_{1}(x, \theta)$ remains constant. However, for atoms in the $|0\rangle$ state the lattice potential depth $V_{0}(x, \theta)$ decreases to $50 \%$ in a lin $\perp$ lin configuration. In order to reduce this effect we tilt the EOM by $3^{\circ}$ and thereby decrease the strength of the $\sigma^{-}$standing wave but increase the strength of the $\sigma^{+}$standing wave in such a polarization configuration. Then both trapping frequencies for the $|0\rangle$ and the $|1\rangle$ state decrease to approx. $85 \%$ in a lin $\perp$ lin configuration relative to their initial value of $\omega=2 \pi \times 45 \mathrm{kHz}$ in a lin $\|$ lin standing wave configuration. For such trapping frequencies of $\approx 45 \mathrm{kHz}$ during the transport process, the excitation probability should remain below $5 \%$ for shifting times longer than $\approx 2 \pi / \omega_{x}$, taking into account the finite bandwidth of our high voltage amplifier. This finite bandwidth smoothes the edges of our linear voltage ramp and thereby efficiently suppresses the oscillatory structure in the calculated excitation probability (see Fig. 22).

In order to verify the coherence of the spin-dependent transport we use the interferometer sequence of Fig. 3 Let us first consider the case of a single atom being initially localized to the $j^{\text {th }}$ lattice site. First, the atom is placed in a coherent superposition of the two internal states $|0\rangle_{j}$ and $|1\rangle_{j}$ with a $\pi / 2$ microwave pulse (here the index denotes the position in the lattice). Then the polarization angle $\theta$ is rotated to $180^{\circ}$, such that the spatial wave packet of an atom in the $|0\rangle$ and the $|1\rangle$ state are transported in opposite directions. The final state after such a movement process is then given by $1 / \sqrt{2}\left(|0\rangle_{j}+i \exp (i \beta)|1\rangle_{j+1}\right)$, where the wave function of an atom has been delocalized over the $j^{\text {th }}$ and the $(j+1)^{t h}$ lattice site. The phase $\beta$ between the two wave packets depends on the accumulated kinetic and potential energy phases in the transport process and in general will be nonzero. In order to reveal the coherence between the two wave packets, we apply a final $\pi / 2$ microwave pulse that erases the which-way information encoded in the hyperfine states. We then release the atoms from the confining potential by suddenly turning off the standing wave optical potential and observe the momentum distribution of the trapped atoms in the $|1\rangle$ state with absorption imaging after a time of flight period. As a result of the above sequence, the spatial wave packet of an atom in the $|0\rangle(|1\rangle)$ state is delocalized over two lattice sites resulting in a double slit momentum distribution $w(p) \propto \exp \left(-p^{2} /\left(\hbar / \sigma_{x}\right)^{2}\right) \cdot \cos ^{2}\left(p \delta x_{0} / 2 \hbar+\beta / 2\right)$ (see Fig. $4 \pi$ ) where $\delta x_{0}$ denotes the separation between the two wave packets and $\sigma_{x}$ is the spatial extension of the gaussian ground state wave function on each lattice site. In order to increase the separation between the two wave packets further, one could increase the polarization angle $\theta$ to further integer multiples of $180^{\circ}$. In practice, such an approach is however limited by the finite maximum voltage that can be applied to the EOM. In order to circumvent this limitation we apply a microwave $\pi$ pulse after the polarization has been rotated to $\theta=+180^{\circ}$, thereby swapping the role of the two hyperfine states. By then returning the polarization vector to $\theta=0^{\circ}$, we do not bring the two wave packets of an atom back to their original site but rather further increase the separation between the wave packets (see Fig. 31). The interlaced $\pi$ 
pulse provides a further advantage of cancelling inhomogeneous phase shifts acquired in the single particle phase $\beta$ in a photon echo like sequence. With increasing separation between the two wave packets the fringe spacing of the interference pattern further decreases (see Fig. (4). We have been able to observe such interference patterns for two wave packets delocalized over up to 7 lattice sites (see Fig. 4f). When moving the atoms over up to three lattice sites, the visibility of the interference pattern remains rather high with up to $60 \%$ (see Fig. 5). These high contrast interference patterns directly prove the coherence of the transport process but also show that the single particle phase $\beta$ acquired for each atom is almost constant throughout the cloud of atoms in our system. If the movement process is repeated more often, inhomogeneously acquired phase shifts over the cloud of atoms significantly decrease the visibility.

For many further applications of the coherent spindependent transport it will also be crucial that the single particle phase $\beta$ is not only constant throughout the cloud of atoms within a single run of the experiment, but is also reproducible between different sets of experiments. We have verified this by varying the phase $\alpha$ of the final microwave $\pi / 2$ pulse in a sequence where an atom is delocalized over three lattice sites. In Figure [ we plot the experimentally measured phase of the interference pattern vs the phase $\alpha$ of the final microwave pulse obtained in different runs of the experiment. We find a high correlation between the detected phase of the interference pattern vs the phase of the applied microwave pulse which proves that indeed the single particle phase is constant between different experiments and can be cancelled via the phase of the final microwave pulse.

In conclusion we have demonstrated the coherent spindependent transport of neutral atoms in optical lattices, thereby showing an essential level of coherent control for many future applications. The method demonstrated here e.g. provides a simple way to continuously tune the interspecies interactions by controlling the overlap of the the two ground state wave functions for the two spin states. Furthermore, if such a transport is carried out in a three dimensional lattice, where the on-site interaction energy between atoms is large, one could induce interactions between almost any two atoms on different lattice sites in a controlled way. Such controlled interactions of Ising or Heisenberg type could then be used to simulate the behavior of quantum magnets 14], to realize quantum gates between different atoms 8, 9, 10, 11] or to generate highly entangled cluster states $[9,12$ that could form the basis of a one-way quantum computer [10].

We would like to thank Ignacio Cirac and Hans Briegel for stimulating discussions, Alexander Altmeyer for help during the initial phase of the experiment and Anton Scheich for assistance with the electronics. We also acknowledge financial support from the Bayerische Forschungsstiftung.

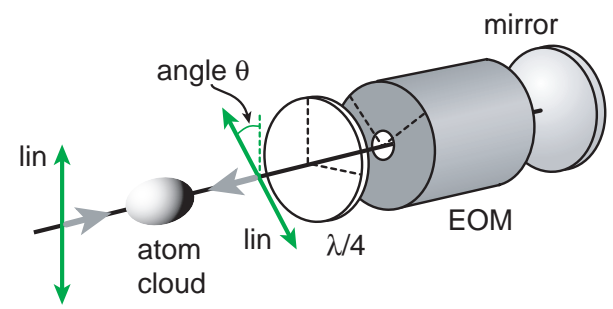

FIG. 1: Schematic experimental setup. A one dimensional optical standing wave laser field is formed by two counterpropagating laser beams with linear polarizations. The polarization angle of the returning laser beam can be adjusted through an electro-optical modulator. The dashed lines indicate the principal axes of the wave plate and the EOM.

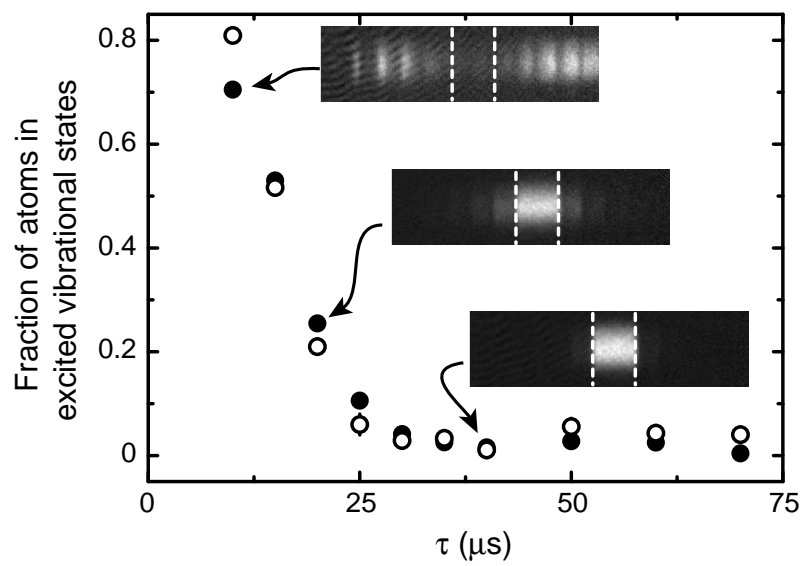

FIG. 2: Fraction of atoms in excited vibrational states after moving the lattice potential in a time $\tau$ over a distance of $\lambda_{x} / 4$. Filled (hollow) circles denote atoms in the $|1\rangle(|0\rangle)$ state. The images show the population of the Brillouin zones when the lattice potential was adiabatically ramped down after the shifting process. These absorption images correspond to the $|1\rangle$ state and were taken after a time of flight period of $14 \mathrm{~ms}$. The white dashed lines in the images denote the borders of the first Brillouin zone. Atoms within this Brillouin zone correspond to atoms in the vibrational ground state on each lattice site.

* Electronic address: imb@mpq.mpg.de URL: http://www.mpq.mpg.de/ haensch/bec

[1] B. P. Anderson and M. A. Kasevich, Science 282, 1686 (1998).

[2] F. S. Cataliotti et al., Science 293, 843 (2001).

[3] D. Jaksch et al., Phys. Rev. Lett. 81, 31081998.

[4] C. Orzel et al., Science 291, 2386 (2001).

[5] M. Greiner et al., Nature 415, 39 (2002).

[6] M. Greiner et al., Nature 419, 51 (2002).

[7] R. Grimm, M. Weidemüller, and Y. B. Ovchinnikov, Adv. At. Mol. Opt. Phys. 42, 95 (2000).

[8] G. K. Brennen et al., Phys. Rev. Lett. 82, 1060 (1999). 


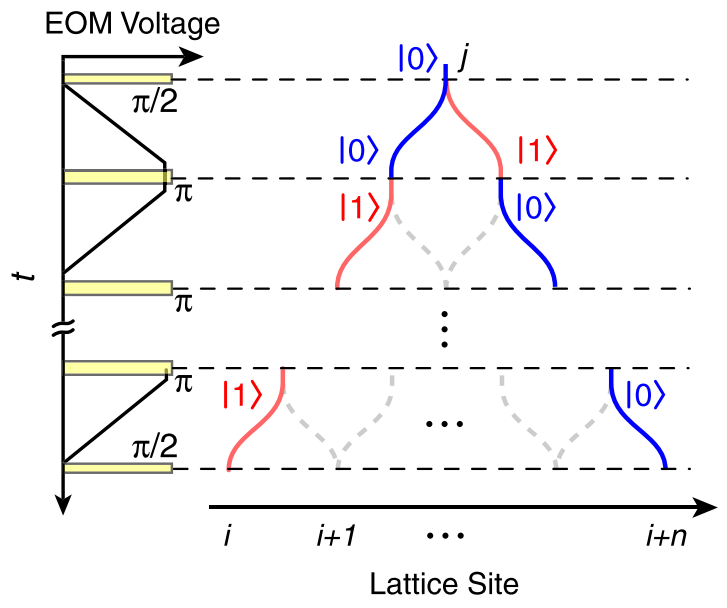

FIG. 3: General interferometer sequence used to delocalize an atom over an arbitrary number of lattice sites. Initially an atom is localized to the $j^{t h}$ lattice site. The graph on the left indicates the EOM voltage and the sequence of $\pi / 2$ and $\pi$ microwave pulses that are applied over time (see text).
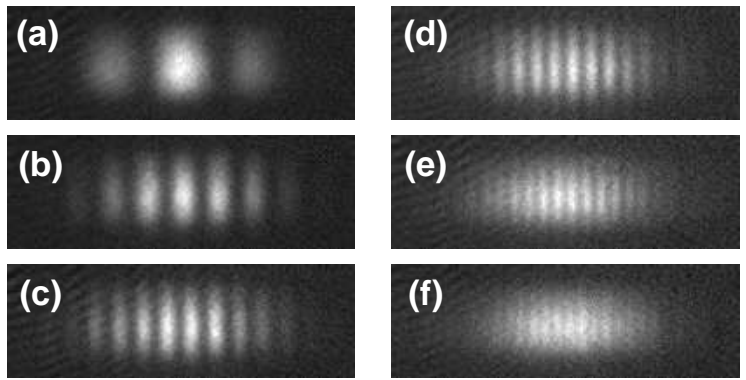

FIG. 4: Observed interference patterns in state $|1\rangle$ after initially localized atoms have been delocalized over (a) two, (b) three, (c) four, (d) five, (e) six and (f) seven lattice sites using the interferometer sequence of Fig. 3 The time of flight period before taking the images was $14 \mathrm{~ms}$ and the horizontal size of each image is $880 \mu \mathrm{m}$.

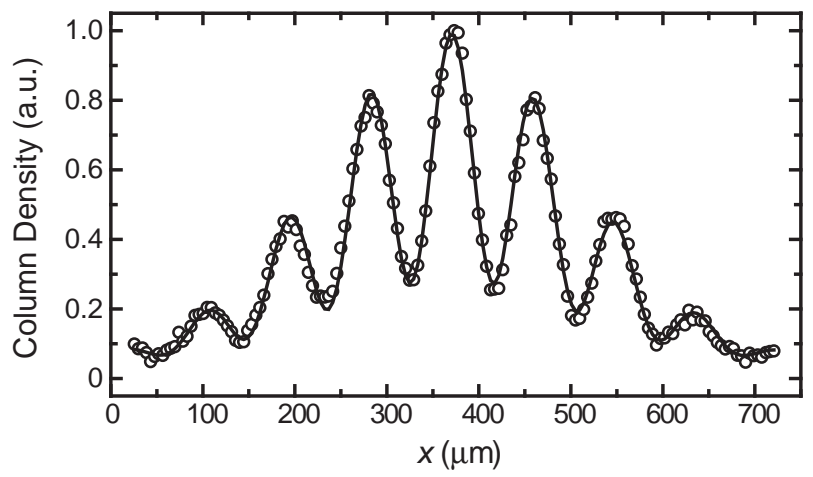

FIG. 5: Profile of the interference pattern obtained after delocalizing atoms over three lattice sites with a $\pi / 2-\pi-\pi / 2$ microwave pulse sequence. The solid line is a fit to the interference pattern with a sinusoidal modulation, a finite visibility $(\approx 60 \%)$ and a gaussian envelope. The time of flight period was $15 \mathrm{~ms}$.

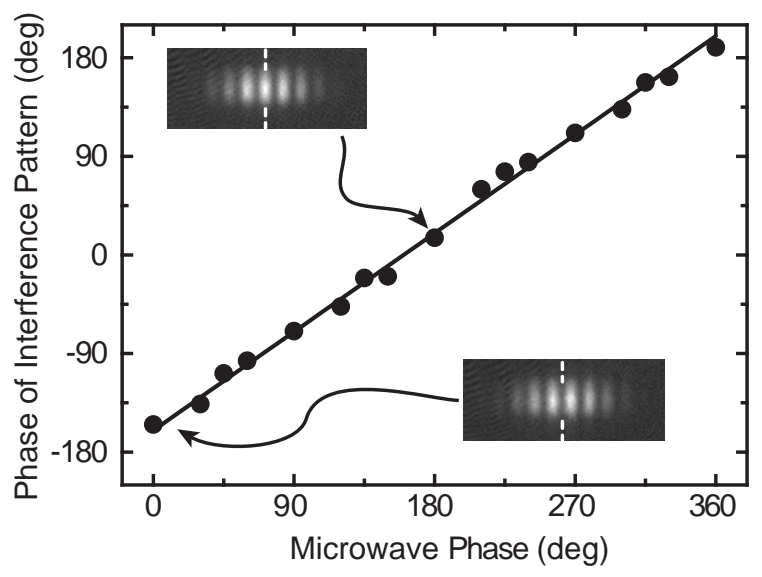

FIG. 6: Phase of the interference pattern vs the phase $\alpha$ of the final microwave $\pi / 2$-pulse in a $\pi / 2-\pi-\pi / 2$ delocalization sequence (see Fig. 3). The absorption images show the measured interference pattern for $\alpha=0^{\circ}$ and $\alpha=180^{\circ}$ after a time of flight period of $15 \mathrm{~ms}$. The solid line is a linear fit to the data with unity slope and a variable offset. The dashed lines in the images correspond to the center of the envelope of the interference pattern.

[9] H.-J. Briegel et al., J. Mod. Opt. 47, 415 (2000).

[10] R. Raussendorf and H.-J. Briegel, Phys. Rev. Lett. 86, 5188 (2001).

[11] G. K. Brennen, I. H. Deutsch, and C. J. Williams, Phys. Rev. A 65, 022313 (2002).

[12] D. Jaksch et al., Phys. Rev. Lett. 82, 1975 (1999).

[13] H.-J. Briegel and R. Raussendorf, Phys. Rev. Lett. 86, 910 (2001).

[14] A. Sørensen and K. Mølmer, Phys. Rev. Lett. 83, 2274 (1999).

[15] W. Dür et al., Phys. Rev. A 66, 052319 (2002).

[16] E. Jané et al., quant-ph/0207011

[17] Spin-dependent transport of neutral atoms has also been observed at NIST. William Phillips, private communication.

[18] D. L. Haycock et al., Phys. Rev. Lett. 85, 3365 (2000).

[19] A. Kastberg et al., Phys. Rev. Lett. 74, 1542 (1999).

[20] M. Greiner et al., Phys. Rev. Lett. 87, 160405 (2001). 